\title{
Impurity in a Luttinger liquid away from half-filling: a numerical study
}

\author{
Shaojin $\mathrm{Qin}^{a}$, Michele Fabrizio ${ }^{b}, \mathrm{Lu} \mathrm{Yu}{ }^{a, c}$ \\ ${ }^{a}$ International Center for Theoretical Physics, P.O. Box 586, 34100 Trieste, Italy. \\ ${ }^{b}$ International School for Advanced Studies, Via Beirut 2-4, I-34014 Trieste, Italy, and Istituto Nazionale di Fisica della \\ Materia, INFM. \\ ${ }^{c}$ Institute of Theoretical Physics, Academia Sinica, Beijing 100080, China.
}

\author{
Masaki Oshikawa $^{d}$, and Ian Affleck ${ }^{d, e}$ \\ ${ }^{d}$ Department of Physics and Astronomy, University of British Columbia, Vancouver BC V6T $1 Z 1$ Canada \\ ${ }^{e}$ Canadian Institute for Advanced Research, University of British Columbia, Vancouver BC V6T $1 Z 1$ Canada
}

(June 17, 2021)

Conformal field theory gives quite detailed predictions for the low energy spectrum and scaling exponents of a massless Luttinger liquid at generic filling in the presence of an impurity. While these predictions were verified for half-filled systems, there was till now no analysis away from this particular filling. Here, we fill in this gap by numerically investigating a quarter-filled system using the density matrix renormalization group technique. Our results confirm conformal field theory predictions, and suggest that they are indeed valid for arbitrary fillings.

PACS numbers: $71.10 . \mathrm{Pm}, 72.15 \mathrm{Nj}$

\section{INTRODUCTION}

The behavior of a one-dimensional (1D) interacting Fermi system (Luttinger liquid) in the presence of a single impurity has been the subject of an intensive theoretical and numerical investigation in recent years, for its interesting anomalies with respect to higher dimensions, and its implications to a variety of physical problems as for instance the behavior of quantum wires (see e.g. Refs. [1,2]) or the tunneling through a constriction in the fractional quantum Hall regime [3]. Specifically, if the interaction is repulsive, the electrons at low energies feel the impurity potential as if it were effectively infinite. On the contrary, for attractive interactions, the effective scattering close to the Fermi energy is vanishingly small (see e.g. Ref. 四). In their seminal paper, Kane and Fisher [A] argued that the low energy behavior for repulsive interaction in fact corresponds to a chain disconnected at the impurity site. In the renormalization group language of boundary conformal field theory (CFT) (see e.g. Refs. [5.6]), for repulsive interaction the open boundary condition (OBC) is the stable fixed point while the periodic boundary condition $(\mathrm{PBC})$ is unstable, and oppositely for attractive interaction (except for the presence of nontrivial fixed points for spinning electrons with spin anisotropic interaction [7] 22]). CFT provides the low energy spectrum and the scaling exponents both for the approach to the appropriate stable fixed point and for the departure from the unstable one.
For a half-filled Luttinger liquid, these predictions have been numerically verified [5, 8]. In the present work, we study numerically the non-half-filled system, which is the generic situation for realistic systems. Compared with the half-filled case, the non-half-filled one has non-zero forward scattering phase shift at the Fermi surface, which makes the analysis more cumbersome. In spite of that, we show that, also away from half-filling, the correct fixed point boundary conditions are those predicted in Refs. 田6.

At the end we will discuss in detail the boundary conditions in relation to some previous studies [9] which gave results different from those mentioned above.

\section{NUMERICAL CALCULATION}

We study numerically the $S=1 / 2$ XXZ Heisenberg chains with anisotropy $a=J_{z} / J_{x y}= \pm 0.5$ at magnetization per site $M=1 / 4$, which correspond to quarter-filled Luttinger liquids with repulsive $(a=0.5)$ or attractive interaction $(a=-0.5)$. We model the impurity by modifying the strength of a particular bond, scaling it by a factor $b$. Specifically, we consider the following Hamiltonian

$$
\begin{aligned}
H= & \sum_{i=1}^{L-1}\left[\left(S_{i}^{+} S_{i+1}^{-}+S_{i}^{-} S_{i+1}^{+}\right) / 2+a S_{i}^{z} S_{i+1}^{z}\right] \\
& +b\left[\left(S_{1}^{+} S_{L}^{-}+S_{1}^{-} S_{L}^{+}\right) / 2+a S_{1}^{z} S_{L}^{z}\right]
\end{aligned}
$$

where $S_{i}^{+}$and $S_{i}^{-}$are spin raising and lowering operators at site $i, S_{i}^{z}$ being its z-component. $L$ is the chain length. $b=0$ and $b=1$ correspond to what we will define as ideal $\mathrm{OBC}$ and $\mathrm{PBC}$, respectively. We use the density matrix renormalization group (DMRG) method [10] to calculate the low energy spectrum and overlaps between ground state wave functions. To ensure the correct magnetization $M=1 / 4$, we insert four sites at each step of DMRG iteration. We run DMRG for $a= \pm 0.5$, $L=8,12, \ldots, 60$, and $b=0.0,0.05,0.1, \ldots, 0.9,0.95,1.0$. The optimal number of states which are kept is 300 and the truncation errors are less than $5 \times 10^{-7}$. Like our previous DMRG calculation for magnetization $M=0$ 
[8], we calculate the lowest energy states with parity even and odd (with respect to the modified bond), and $z$ component of total spin $S_{z}^{\text {tot }}=M L$ and $M L+1$ for $M=1 / 4$. We denote these energies in increasing order as $E_{0}, E_{1}, E_{2}$, and $E_{3}$. For states with magnetization $M$, $E_{0}$ corresponds to the ground state energy while $E_{2}$ corresponds to the energy of the lowest excited state with different parity. $E_{1}$ and $E_{3}$ correspond to states with $S_{z}^{t o t}=M L+1$, but with different parity. In addition to these low energy levels, we calculate also the ground state wave function overlaps between PBC chains $(b=1)$ and impurity chains $(b \neq 1)$.

\section{ENERGY SPECTRUM AND SCALING PREDICTED IN CFT}

The spin chain at non-zero $M$ and with a modified bond can be written in terms of a boson field, $\phi$, which is defined periodically: $\phi \equiv \phi+2 \pi R$, and of its conjugate momentum $\Pi$. A detailed derivation is given in Ref. [5] for the case $M=0$. Here we just point out the differences that occur for non-zero $M$. The compactification radius, $R$, and the spin-wave velocity, $v_{s}$, depend both on $a$ and on $M$. Their values for $a= \pm 0.5$ and $M=1 / 4$ are given in Table I. The effective Hamiltonian is:

$\mathcal{H}=\int d x \frac{1}{2}\left[\Pi^{2}+\left(\frac{d \phi}{d x}\right)^{2}\right]-\delta(x)\left[V_{f} \frac{d \phi}{d x}+V_{b} \cos \left(\frac{\phi}{R}\right)\right]$.

[We will generally set the spin-wave velocity $v_{s}$ to 1 in most of what follows.] Here the modified bond is parameterized in terms of two boundary operators, with coupling constants $V_{f}$ and $V_{b}$. The $V_{f}$ term is allowed, and therefore expected to occur, whenever $S^{z} \rightarrow-S^{z}$ symmetry as well as translational invariance are broken, which is the case for $M \neq 0$ and with a modified bond. Specifically, $V_{f}$ and $V_{b}$ correspond to forward and backward scattering in the Luttinger liquid, respectively. Forward scattering is marginal but backward scattering is relevant (irrelevant) for $R>1 / \sqrt{4 \pi}(R<1 / \sqrt{4 \pi})$.

In cases where backscattering is irrelevant, which we define generically as PBC cases (not to be confused with ideal $\mathrm{PBC}$ with $b=1), V_{f}$ induces a discontinuity in $\phi$ at the impurity site, $\phi(0+)-\phi(0-) \equiv \phi(0)-\phi(L)=$ $2 R \delta$ modulus $2 \pi R$. Therefore the mode expansion can be written as:

$$
\phi(x, t)=2 \pi R\left(n-\frac{\delta}{\pi}\right) \frac{x}{L}+\frac{m t}{R L}+\ldots
$$

Here the dots represent the harmonic oscillator modes. $n=S_{z}^{\text {tot }}-M L$ and $m$ are integer quantum numbers. (The $\phi$ periodicity is preserved in time by the discrete quantization of the momentum conjugate.) Thus the finite-size spectrum is:

$$
\begin{aligned}
E= & e_{0} L+e_{1}+n \mu+\frac{2 \pi v_{s}}{L}\left[-\frac{1}{12}+\pi R^{2}\left(n-\frac{\delta}{\pi}\right)^{2}\right. \\
& \left.+\frac{m^{2}}{4 \pi R^{2}}+\sum_{n=1}^{\infty} n\left(m_{n}^{L}+m_{n}^{R}\right)\right] .
\end{aligned}
$$

$m_{n}^{L, R}$ are occupation numbers for bosonic excitations of momentum $\pm 2 \pi n / L$. The chemical potential, $\mu$, is related to the external magnetic field necessary to induce the magnetization, $M$, in the spin chain. $\delta$ is the analogous to a forward scattering phase shift. Here we have included the zero point energy $-2 \pi v_{s} /(12 L)$ which was ignored in Ref. [5]. This formula differs slightly from the one in Ref. [6] because in that case spinless fermions were considered rather than $S=1 / 2$ spins. While the models are identical under the Jordan-Wigner transformation, $\mathrm{PBC}$ on the spins do not correspond to $\mathrm{PBC}$ on the fermions.

When the backscattering term is relevant, $\phi(0)$ gets pinned. In analogy with the previous case, we define this situation as $\mathrm{OBC}$, again not to be confused with the ideal OBC $(b=0)$. Without forward scattering (for $M=0$ ) $\phi(0)$ will simply be pinned at a unique value, $\phi(0)=0$ assuming $V_{b}>0$. With both backward and forward scattering present, we expect $\phi$ to be pinned at equal and opposite values, $\pm \phi_{0}$ at $x \rightarrow 0^{ \pm}$, with a discontinuity at $x=0$. Pinning forces $m=0$ in the mode expansion, changes the wavefunctions of the harmonic modes from $e^{ \pm i 2 \pi n x / L}$ to $\sin (\pi n x / L)$ and reduces the zero point energy by a factor of $1 / 4$. Thus

$$
\begin{aligned}
E= & e_{0} L+e_{1}+n \mu+\frac{\pi v_{s}}{L}\left[-\frac{1}{24}+2 \pi R^{2}\left(n-\frac{\delta}{\pi}\right)^{2}\right. \\
& \left.+\sum_{n=1}^{\infty} n m_{n}\right] .
\end{aligned}
$$

The parameters $e_{0}, v_{s}, R$ and $\mu$ being bulk properties are the same for $\mathrm{PBC}$ and $\mathrm{OBC}$ cases. The parameters $e_{1}$ and $\delta$, on the other hand, depend on the boundary conditions, as well as the $1 / 12$ and $1 / 24$ terms. The latter can be calculated by elementary methods for the $\mathrm{XX}$ spin chain with $\mathrm{PBC}$ or OBC, respectively. They have also been calculated, using the Bethe ansatz for the general XXZ model. They follow more generally from conformal invariance. [11,12 Note that forward scattering always introduces a discontinuity in $\phi$ at the origin, with or without pinning of $\phi$ depending on whether or not backscattering is relevant. However, we expect the value of the discontinuity $\propto \delta$ to depend on whether or not pinning occurs.

The effect of a parity transformation on the various eigenstates were worked out in Ref. [5]. In the periodic case it takes $m \rightarrow-m, m_{n}^{L} \leftrightarrow m_{N}^{R}$ and multiplies wavefunctions by $(-1)^{n+m}$. In the case of OBC it multiples wavefunctions by $(-1)^{n}$ and transform a bosonic operator $a_{m}$, corresponding to an excitation with wave function $\sin (\pi m x / L)$, into $(-1)^{m} a_{m}$. 
We are now in position to work out the quantum numbers and energies of the four lowest energy states. Consider first the states with $S_{z}^{t o t}=M L$. These states have $n=0$. The ground state has all other quantum numbers 0 as well. For PBC, a state of reversed parity can be obtained either by taking $m=1$ (and all other quantum numbers 0) in Eq. (3.3) or by taking an odd parity linear combination of the states with $\left(m_{1}^{L}, m_{1}^{R}\right)=(1,0)$ or $(0,1)$ (and all other quantum numbers 0 ). These two states have energies measured from the ground state energy of $\left(2 \pi v_{s} / L\right) /\left(4 \pi R^{2}\right)$ and $\left(2 \pi v_{s} / L\right)$ respectively. Thus which of these states has lower energy depends on whether $4 \pi R^{2}>1$ or $4 \pi R^{2}<1$. We see from Table I that the first one has lower energy for $a=1 / 2$, while the second one has lower energy for $a=-1 / 2$. The states with $S_{z}^{\text {tot }}=M L+1$ have $n=1$. The state with all other quantum numbers 0 has reversed parity relative to the grounds state. For PBC the state with the same parity as the ground state is obtained again by choosing $m=1$ (for $4 \pi R^{2}>1$ ) or either $m_{1}^{L}$ or $m_{1}^{R}=1$ (for $4 \pi R^{2}<1$ ). For OBC, the lowest energy states for given $S_{z}^{\text {tot }}$ (i.e. $n$ ) are obtained by choosing all the $m_{n}=0$, while the parity reversed lowest energy states are obtained by choosing $m_{1}=1$. We define new quantities, $d_{i}$, by writing the $1 / L$ term in the energy as

$$
E_{i}=\ldots+\frac{2 \pi v_{s}}{L}\left(-\frac{1}{12}+d_{i}\right)
$$

for $\mathrm{PBC}$ or $\mathrm{OBC}$. The values of the $d_{i}$ for the first four states for ideal PBC and OBC for $a= \pm 0.5$ are given in Table II.

The renormalization group flow in the vicinity of the $\mathrm{PBC}$ and $\mathrm{OBC}$ fixed points are determined by the permitted boundary operator, $\mathcal{O}$, of lowest scaling dimension, $x$. Depending on whether this dimension obeys $x<1$ or $x>1$, the fixed point will be unstable or stable. We may calculate the (size dependent) corrections to the energies, in the vicinity of the fixed point, by doing lowest order perturbation theory in this operator. 13 The dimensions of a boundary operator at the PBC and OBC fixed points can be read off from the finite size energies. Specifically, if a boundary operator modifies some quantum numbers, its dimension is simply given by the term inside the square brackets of Eq. (3.3) for PBC and (3.4) for $\mathrm{OBC}$ with the appropriate change of quantum numbers, minus the analogous term with the ground state quantum numbers.

For instance, the leading boundary operator which can be defined at the PBC fixed point is the backscattering potential $\mathcal{O}=\cos (\phi / R)$ [see the Hamiltonian (3.1)]. Since it changes $m$ by \pm 1 , its scaling dimension is simply $x=1 / 4 \pi R^{2}$. The lowest order perturbative calculation is quite different depending on whether $4 \pi R^{2}>1$ or $<1$. Let us denote $\mid 0>$ and $\mid 2>$ the states corresponding to the energy levels $E_{0}$ and $E_{2}$ previously introduced, and use $d_{0}$ and $d_{2}$ defined in Eq.(3.5). If $4 \pi R^{2}>1$, then $\mathcal{O}$ is relevant and the $\mathrm{PBC}$ fixed point is unstable. In this case the correction to $d_{2}-d_{0}$ becomes larger than the fixed point value, at sufficiently large $L$. Since the state $|2\rangle$ has $m=1$, and $m$ corresponds to a conserved quantum number at the $\mathrm{PBC}$ fixed point, the only non-zero matrix element of $\cos (\phi / R)$ in the subspace of $\mid 0>$ and $\mid 2>$ is an off-diagonal one $<2|\cos (\phi / R)| 0>$. Since this is larger than the diagonal matrix elements, the energy difference, divided by $L$, scales as $L^{y}$, where $y$ is the renormalization group eigenvalue of the associated coupling constant, $y \equiv 1-x$. On the other hand, for $4 \pi R^{2}<1$, the PBC fixed point is stable, and in fact the corrections to the $d_{i}$ 's from $\mathcal{O}$ vanish at large $L$. In this case, the state $\mid 2>$ has $m=0$ (and $m_{1}^{L}$ or $m_{1}^{R}=1$ ). Hence the first order corrections to the energies vanish and the leading corrections are second order, that is $d_{2}-d_{0} \propto L^{2 y}$.

At the OBC fixed point, the most relevant permitted operator is a product of the operators which change $n \pm 1$ from the two sides of the broken chain, that is $S_{1}^{+} S_{L}^{-}+$h.c.. The $n= \pm 1$ operator has dimension $2 \pi R^{2}(1 \mp 2 \delta / \pi)$. Since the two sides of the broken chain are decoupled at the ideal OBC fixed point, the dimension of the product of operators on the two sides is simply additive, giving a total dimension for the leading operator of $4 \pi R^{2}$. Since there is no extra conserved quantum number at the OBC fixed point we expect this operator to have a non-vanishing expectation value in the states $\mid 0>$ and $\mid 2>$. Hence the correction to $d_{2}-d_{0}$ should scale as $L^{y}$.

Exactly at $b=0$ or 1 , the set of allowed operators is reduced. For the PBC case, $b=1$, all local as well as $m \neq 0$ operators are forbidden by translation invariance. The leading irrelevant operators correspond to

$$
\int d x(\partial \phi / \partial x)(\partial \phi / \partial x)^{2} \quad \int d x(\partial \phi / \partial x)(\partial \phi / \partial t)^{2}
$$

of dimension 2. $[\partial \phi / \partial x$ can be eliminated by a redefinition of $\phi$.] These dimension 2 operators are forbidden by symmetry for $M=0$ and so did not appear in [5]. They contribute $1 / L^{2}$ corrections to the energies. For the $b=0$ case, since translational symmetry is broken, we can construct boundary operators but only at one side or the other of the broken link; we can not take a product of operators on both sides as above. In addition, spin rotational invariance forbids all operators with $n \neq 0$. Again $\partial \phi / \partial x$ is eliminated by a redefinition of $\phi$, so the leading irrelevant boundary operator is $(\partial \phi(0) / \partial x)^{2}$ of dimension 2 [5]. This also contributes $1 / L^{2}$ corrections to the energies.

When $b$ is close to 0 or 1 , we obtain analytic $1 / L^{2}$ finite-size corrections, as just discussed, with coefficients of $O(1)$, as well as non-analytic finite size corrections, as previously shown, with coefficients that vanish in the limit $b \rightarrow 0$ or 1 . 


\section{NUMERICAL STUDIES ON SPECTRUM AND RENORMALIZATION}

We first consider the bulk quantities, $e_{0}, \mu, v_{s}$ and $R$, defined in Eq. (3.3). We determined these using both the DMRG and the Bethe ansatz technique 16.

These bulk quantities can be determined from the finite-size spectrum with $\mathrm{PBC}$ which can be derived from the Bethe ansatz [17]. The structure of the spectrum up to $O(1 / L)$ term agrees with the CFT prediction $\left[\delta=0, e_{1}=0\right.$ in Eq. (3.3) ]. By comparison, the various parameters are determined [17] as:

$$
\begin{aligned}
e_{0} & =\int_{-\Lambda}^{\Lambda} \epsilon_{0}(\eta) \sigma(\eta) d \eta \\
R & =\frac{1}{\sqrt{4 \pi} \xi(\Lambda)} \\
v_{s} & =\frac{e}{2 \pi \sigma(\Lambda)}
\end{aligned}
$$

Here the right-hand sides are given by

$$
\begin{aligned}
\epsilon_{0}(\eta)= & \mu-\frac{\sin ^{2} \theta}{\cosh \eta-\cos \theta}, \\
\xi(\eta)= & 1-\frac{1}{2 \pi} \int_{-\Lambda}^{\Lambda} K\left(\eta-\eta^{\prime}\right) \xi\left(\eta^{\prime}\right) d \eta^{\prime} \\
\sigma(\eta)= & \frac{1}{2 \pi}\left[\frac{\cot (\theta / 2)}{\cosh ^{2}(\eta / 2)+\cot ^{2}(\theta / 2) \sinh ^{2}(\eta / 2)}\right. \\
& \left.-\int_{-\Lambda}^{\Lambda} K\left(\eta-\eta^{\prime}\right) \sigma\left(\eta^{\prime}\right) d \eta^{\prime}\right], \\
\rho(\eta)= & \frac{1}{2 \pi}\left[\frac{d K(\eta-\Lambda)}{d \eta}-\int_{-\Lambda}^{\Lambda} K\left(\eta-\eta^{\prime}\right) \rho\left(\eta^{\prime}\right) d \eta^{\prime}\right] \\
e= & \frac{d \epsilon_{0}(\Lambda)}{d \Lambda}+\int_{-\Lambda}^{\Lambda} \epsilon_{0}(\eta) \rho(\eta) d \eta
\end{aligned}
$$

where $\cos \theta=a$ (see Eq. 2.1)) and the integral kernel is given by

$$
K(\eta)=\frac{\tan \theta}{\tan ^{2} \theta \cosh ^{2}(\eta / 2)+\sinh ^{2}(\eta / 2)} .
$$

The parameter $\Lambda$ is fixed from the filling factor $\nu=1 / 4$ by

$$
\int_{-\Lambda}^{\Lambda} \sigma(\eta) d \eta=\nu
$$

and the chemical potential $\mu$ is determined by the condition $\epsilon_{d}(\Lambda)=0$, where

$$
\epsilon_{d}(\eta)=\epsilon_{0}(\eta)-\frac{1}{2 \pi} \int_{-\Lambda}^{\Lambda} K\left(\eta-\eta^{\prime}\right) \epsilon_{d}\left(\eta^{\prime}\right) d \eta^{\prime}
$$

The half-filled case $\nu=1 / 2$ corresponds to $\Lambda=\infty$. In this case the integral equations can be solved analytically by Wiener-Hopf method [17], which gives $R=\sqrt{\frac{1-\theta / \pi}{2 \pi}}$ and $v_{s}=\pi \sin \theta /(2 \theta)$, as deduced earlier from the Bethe ansatz solution using other methods. [18,19] In particular, for $a= \pm 0.5$ and $M=0$, the case considered in Ref. [8], $v_{s}=3 \sqrt{3} / 4,3 \sqrt{3} / 8$, and $4 \pi R^{2}=2 / 3,1 / 3$, respectively. The Bethe ansatz results for $L\left(E_{1}-E_{0}\right)$, $e_{2} \equiv\left(E_{2}-E_{0}\right) /\left(E_{1}-E_{0}\right)$ and $e_{3} \equiv\left(E_{3}-E_{0}\right) /\left(E_{1}-E_{0}\right)$ are given in Table III. Excellent agreement is obtained with the finite-size spectrum calculated using DMRG in 8.

For the non-half-filled case, these equations cannot be solved analytically. We solved them numerically by iterative numerical integration. Since we have already taken the limit of large system size $L$ in analytic treatment, we can obtain highly accurate results with relatively small amount of computation. Accuracy to six digits can be achieved within minutes on an IBM RS6000 workstation. On the other hand, it would be difficult to calculate other quantities, for example orthogonality exponent, directly from Bethe Ansatz.

By fitting all four $E_{i}$ 's obtained from DMRG according to Eqs. (3.3) and (3.4), for the cases we defined as ideal $\mathrm{PBC}(b=1)$ and $\mathrm{OBC}(b=0)$, we find consistent bulk quantities, $e_{0}, \mu, v_{s}$, and $R$, which are listed in Table I. These values agree well with the Bethe Ansatz results, as also shown in Table I. Thus the accuracy of our DMRG calculation is confirmed. We also determined, from DMRG, the non-zero forward scattering phase shift, $\delta$, for the case of OBC, as shown in Table I.

With these parameters, we have found that the finitesize corrections to the energies, $d_{i}$, found numerically agree with the low energy spectrum predicted by CFT for PBC and $\mathrm{OBC}$ and listed in Table II. The finite size values of $d_{i}$ are plotted versus $1 / L$ in Figures 1 and 2, and extrapolated at $L \rightarrow \infty$ by fitting with polynomials of $1 / L$. The quality of these fittings confirms the prediction that only analytic finite size corrections exist in the cases with $b=0$ or 1 .

After having verified the low energy CFT spectrum, we confirm another prediction, namely that the orthogonality exponent between the ground states of two models described by the same Hamiltonian but different conformally invariant boundary conditions, is equal to the difference between their corresponding $d_{0}$ 's. The orthogonality exponent $\alpha$ is defined through the overlap between the two ground state wave functions, $|\phi\rangle$ and $\left|\phi_{0}\right\rangle$, for a chain of size $L$. In particular,

$$
O(L)=\left\langle\phi \mid \phi_{0}\right\rangle \propto\left(\frac{1}{L}\right)^{\alpha}
$$

The overlap integrals $O(L)$ are evaluated numerically. The $\alpha$ for $L \rightarrow \infty$ limit is obtained by extrapolating

$$
\alpha(L)=\frac{\ln O(L+4)-\ln O(L)}{\ln L-\ln (L+4)} .
$$

We start by verifying the above prediction for the overlap between the ground states with ideal PBC and OBC. 
The ideal OBC $d_{0}$ 's for $L \rightarrow \infty$ limit (for the ideal PBC, $\left.d_{0}=0\right)$ is obtained by extrapolating

$$
d_{0}(L)=\frac{1}{12}-\frac{L(L+4)}{8 \pi v_{s}}\left[E_{0}(L+4)-E_{0}(L)-4 e_{0}\right] .
$$

In Fig. 3 we plot $\alpha(L)$, the exponent of the overlap between the ideal $\mathrm{OBC}$ and $\mathrm{PBC}$ ground state wavefunctions, and the ideal $\mathrm{OBC} d_{0}(L)$, vs. $1 / L$. Up to the two significant digits the extrapolated $\alpha$ and $d_{0}$ are equal for $L \rightarrow \infty$ limit, for both $a=0.5$ and $a=-0.5$.

We point out here that the low energy spectrum in Table II holds also if the magnetization $M=0$, as it was shown in Ref. [8]. For generic impurity strength, it was shown in that reference that the $M=0$ system flows either to OBC $(a>0)$ or to PBC $(a<0)$, by a detailed analysis of the size dependence of the low energy levels and by calculating the orthogonality exponent. Since the non-universal forward scattering phase shift $\delta=0$ for $M=0$, universality is recovered and all systems flowing to the OBC fixed point had an extrapolated $d_{0}=1 / 16$, in agreement with CFT prediction. Moreover, 1/16 was also shown to be equal to the orthogonality exponent of the overlap between the ground states with $\mathrm{PBC}$ in the presence and in the absence of the impurity.

Analogously, in the following, we will first show that the low energy spectrum for $M=1 / 4$ systems does flow to the $\mathrm{PBC}$ or OBC fixed points, depending on the sign of the interaction. Then we will show the qualitative agreement between $\alpha(L)$ and $d_{0}(L)$ for arbitrary impurity strength $b$ at finite size.

We start by showing how the low energy spectrum flows away from PBC towards the OBC fixed point for $a=0.5$ and vice versa for $a=-0.5$. We previously said that $\delta$ is not a universal property. This implies that, if a system flows to one of the two fixed points, it does not mean necessarily that the value of $\delta$ will be exactly the same as that of a chain with $b=0$ or $b=1$ (as given in Table I). Thus we expect $\delta$ to be some non-universal function of $b$ and $a$. The finite size spectrum should be given by the OBC formula of Eq. (3.4) and Table II for $a=0.5$ and all $b$ but with some unknown function $\delta(b)$. Likewise, for $a=-0.5$ the spectrum should be given by the PBC formula of Eq. (3.3) and Table II for all $b$ but with some other unknown function $\delta(b)$. $\delta(b)$ should approach zero as $b \rightarrow 1$ for $a=-0.5$ and $\delta(b)$ should approach its OBC value $(\approx-0.29 \pi)$ as $b \rightarrow 0$ for $a=0.5$ since in these two cases $1-b$ or $b$ produce no relevant operators and the marginal operator has a coupling constant which goes to zero.

For the four energy levels we decided to calculate numerically, $E_{3}-E_{1}=E_{2}-E_{0}$ always holds, as seen from Table II. Notice that the forward scattering phase shift $\delta$ generated by the impurity contributes to $E_{1}-E_{0}$ (and also $E_{3}-E_{2}$ ). In order to simplify the analysis, we will concentrate only on $E_{2}-E_{0}$ (i.e. $\left.d_{2}-d_{0}\right)$, which instead depends on the universal properties identifying each fixed point. Let us denote $\Delta(a, b)=d_{2}(a, b)-d_{0}(a, b)$. Then, for small deviations of $b$ away from one of the two fixed point values $b_{*}=1,0$, the following scaling should hold

$$
\Delta(a, b)-\Delta\left(a, b_{*}\right) \propto L^{\gamma\left(a, b_{*}\right)} .
$$

These exponents can be obtained by calculating the first non-vanishing correction to $\Delta(a, b)$, within a perturbation expansion in $b-b_{*}$, following the prescriptions given in the previous section. The predicted $\gamma$ 's are listed in Table IV. In Fig.4, for $a= \pm 0.5$, we plot $\ln \left(\Delta(a, b)-\Delta\left(a, b_{*}\right)\right) / \ln L$ vs. $1 / \ln L$. The extrapolated scaling exponents $\gamma$ in the figure agree with those listed in Table IV, up to the two significant digits. These results have a very simple interpretation. If $\gamma<0$, the deviation from the fixed point is not a relevant perturbation and the system flows back to the $b_{*}$-fixed point, which is therefore stable. On the contrary, if $\gamma>0$, the perturbative correction blows up as $L \rightarrow \infty$, which implies that the system flows away from the $b_{*}$ fixed point, which is therefore unstable. Again, our results demonstrate that, for repulsive interactions, the stable fixed point boundary condition is the $\mathrm{OBC}$, and vice versa for attractive interaction.

We have also calculated $\Delta(a, b)$ for arbitrary deviations $b-b_{*}$, namely for $b=0.05,0.15, \ldots, 0.85,0.95$. To analyze the data, we made a very simple scaling ansatz in order just to reproduce the correct scaling behavior close to the OBC fixed point. Specifically, $\Delta(a, b)$ is plotted in Fig.5 vs. $\arctan \left[L^{-0.2} \tan \left(b \frac{\pi}{2}\right)\right] / \pi$ for $a=0.5$, and in Fig. 6 vs. $\arctan \left[L^{0.28} \tan \left(b \frac{\pi}{2}\right)\right] / \pi$ for $a=-0.5$. The arrows in the figures show towards which fixed point the system flows as the chain length $L$ increases. We see qualitatively how the excitation energy flows between $\mathrm{OBC}$ and $\mathrm{PBC}$ fixed points. The proposed scaling is $\tan \left[\left(\Delta-\frac{1}{2}\right) \pi\right] \approx L^{\gamma} \tan \left(b \frac{\pi}{2}\right)$. Surprisingly, our scaling ansatz, which is in principle valid only close to $b=0$ and $L^{\gamma}$ being small, seems to give a good description of the data for a wide range of $b$ 's. For $\frac{b}{2} L^{\gamma} \rightarrow 0$, we have the scaling relation:

$$
\Delta-\frac{1}{2} \rightarrow \frac{b}{2} L^{\gamma}
$$

Finally we compare the orthogonality exponent $\alpha(L)$ of the overlap between the ground state with PBC and in the presence of the impurity, and the ground state with $\mathrm{PBC}$ but in the absence of the impurity. According to CFT, this exponent should also be equal to $d_{0}$ for chain with impurity. We plot $\alpha(L)$ and $d_{0}(L)$ vs. the same variables as used above for $\Delta$. The resulting data are plotted in Fig. 7 for $a=0.5$, and in Fig. 8 for $a=-0.5$. They show that $\alpha(L)$ and $d_{0}(L)$ are of similar magnitude and have similar flows for various chain lengths and impurity strengths. Again, an important remark is needed regarding the forward scattering phase shift. Since we can not exclude its generation at the fixed point, this would imply, for instance, that the orthogonality exponent for attractive interaction is, in general, non-zero. With long 
enough chains it should be possible to determine $\delta(b)$ and check that both $\alpha(L)$ and $d_{0}(L)$ extrapolate to the same numbers, determined by these phase shifts. However, with the limited lengths available $(L \leq 60)$ we have not found it possible to do this. This is presumably due to the unknown value of the phase shift, the slow convergence of the finite-size corrections due to the small exponent in the non-analytic corrections $\left(L^{-0.2}\right.$ for $\left.a=0.5\right)$ and the relatively large coefficient in the analytic corrections. The situation appeared somewhat better in the $M=0$ case [8].

\section{DISCUSSION ON BOUNDARY CONDITION}

With periodic boundary conditions, the boson may be written in terms of decoupled left and right-moving parts:

$$
\phi(t, x)=\phi_{L}(t+x)+\phi_{R}(t-x) .
$$

The dual field is defined as $\tilde{\phi} \equiv \phi_{L}-\phi_{R}$. The boundary condition, $\phi\left(0^{ \pm}\right)= \pm \phi_{0}$ couples left movers to right movers, separately on both sides of the origin:

$$
\begin{aligned}
& \phi_{L}\left(t, 0^{-}\right)=-\phi_{R}\left(t, 0^{-}\right)-\phi_{0} \\
& \phi_{R}\left(t, 0^{+}\right)=-\phi_{L}\left(t, 0^{+}\right)+\phi_{0} .
\end{aligned}
$$

On the other hand the fields on opposite sides of the origin are not coupled by the boundary condition. This means that an incoming excitation at $x<0$ is reflected, with unit probability with a phase shift proportional to $\phi_{0}$ and similarly for an incoming excitation at $x>0$. This boundary condition is the same as the one used in Ref. [6] where a different basis was used.

This coupling of left and right movers by relevant backscattering changes Green's functions. [5] To illustrate this, we consider the $M=0$ case where there is no forward scattering term, $\phi_{0}=0$. One term in the bosonized representation of $S_{j}^{-}$is:

$$
\begin{aligned}
S_{j}^{-} & \propto e^{i 2 \pi R \tilde{\phi}+i \phi / R}+\ldots \\
& \propto e^{i(2 \pi R+1 / R) \phi_{L}} e^{i(-2 \pi R+1 / R) \phi_{R}}+\ldots
\end{aligned}
$$

The Green's function, with PBC factorizes into separate Green's functions for left and right movers, giving:

$$
\begin{aligned}
\left\langle S^{+}(t, x) S^{-}\left(0, x^{\prime}\right)\right\rangle \propto & \left(t+x-x^{\prime}\right)^{-(2 \pi R+1 / R)^{2} / 4 \pi} \\
& \times\left(t-x+x^{\prime}\right)^{-(-2 \pi R+1 / R)^{2} / 4 \pi}+\ldots
\end{aligned}
$$

On the other hand, with the perfectly reflecting boundary condition, this correlation function equals 0 if $x$ and $x^{\prime}$ are on opposite sides of the origin. To calculate the Green's function when they are on the same side (say $x, x^{\prime}>0$ ) we use a standard device from boundary CFT. 114 We can regard the second boundary condition of Eq. (5.2) as defining $\phi_{R}$ for all $x>0$ as the analytic continuation of $\phi_{L}$ to the negative $x$-axis:

$$
\phi_{L}(t,-x) \equiv-\phi_{R}(t, x)+\phi_{0} .
$$

The reason this works is because the boundary condition is true at all $t$ and $\phi_{L}$ and $\phi_{R}$ only depend on the variables $t+x$ and $t-x$, respectively. We emphasize that this analytically continued $\phi_{L}$ is not the original $\phi_{L}$ at $x<0$ which is completely decorrelated from the fields at $x>0$. Using this approach, $S^{-}(t, x)$ becomes bilocal:

$$
S^{-}(t, x) \propto e^{i(2 \pi R+1 / R) \phi_{L}(t+x)-i(-2 \pi R+1 / R) \phi_{L}(t-x)}+\ldots
$$

Instead of getting a product of 2-point Green's functions for left and right moving fields, we now obtain a 4-point Green's function for left-movers only, giving:

$$
\begin{aligned}
\left\langle S^{+}(t, x) S^{-}\left(0, x^{\prime}\right)\right\rangle \propto & \left|\frac{x x^{\prime}}{t^{2}-\left(x+x^{\prime}\right)^{2}}\right|^{-1 / 4 \pi R^{2}+\pi R^{2}} \\
& \times\left|\frac{1}{t^{2}-\left(x-x^{\prime}\right)^{2}}\right|^{1 / 4 \pi R^{2}+\pi R^{2}} \\
& \times\left|\frac{t-x+x^{\prime}}{t+x-x^{\prime}}\right|+\ldots
\end{aligned}
$$

In the limit, $x, x^{\prime} \rightarrow 0^{+}$,

$$
S^{-}(t, 0) \propto e^{i 2 \pi R \tilde{\phi}\left(t, 0^{+}\right)} \propto e^{i 4 \pi R \phi_{L}\left(t, 0^{+}\right)},
$$

giving:

$$
<S^{+}\left(t, 0^{+}\right) S^{-}\left(0,0^{+}\right)>\propto|t|^{-4 \pi R^{2}}+\ldots,
$$

which is the limiting behaviour of Eq. (5.7) (with $x x^{\prime}$ set equal to a value of order of the short distance cutoff, i.e. the lattice spacing). Note that without pinning :

$$
\begin{aligned}
& \left\langle e^{i 2 \pi R \tilde{\phi}(t, 0)} e^{-i 2 \pi R \tilde{\phi}(0,0)}\right\rangle \\
& =\left\langle e^{i 2 \pi R \phi_{L}(t, 0)} e^{-i 2 \pi R \phi_{L}(0,0)}\right\rangle\left\langle e^{-i 2 \pi R \phi_{R}(t, 0)} e^{i 2 \pi R \phi_{R}(0,0)}\right\rangle \\
& \propto|t|^{-2 \pi R^{2}} .
\end{aligned}
$$

Thus pinning increases the exponent by a factor of 2 . Perhaps surprisingly, pinning $\phi(0)$ changes correlation functions involving $\tilde{\phi}$. This effect ultimately arises because $\phi$ and $\tilde{\phi}$ cannot be regarded as being independent. $\partial \tilde{\phi} / \partial x$ is canonically conjugate to $\phi$. Therefore when $\phi$ gets pinned we must take into account the effect on $\tilde{\phi}$. Different results were obtained in Ref. [9] because this effect was not taken into account. In particular, a factor of two discrepancy in the exponent occurring in the electron Green's function (at $x=x^{\prime}=0$ ) arose from the mechanism explained here. An alternative way of understanding the disagreement with Ref. [9] can be found in Ref. 20, and in Ref. 21.

\section{SUMMARY}

In summary, this paper studies the effects of an impurity in a non-half-filled 1D Luttinger liquid. Our numerical study for the low energy spectrum confirms previous 
results. That is, we show that the low energy spectrum of a chain with an impurity flows to the spectrum of a chain with $\mathrm{PBC}$ if the interaction is attractive, and to the spectrum of a chain with OBC if the interaction is repulsive. The behavior of a non-half-filled system is shown to be the same as that of a half-filled one and the presence of particle-hole symmetry is not essential for this behaviour. Therefore, we numerically confirm that the properties of a generic 1D Luttinger liquid with an impurity are indeed those predicted by Kane and Fisher 4 .

\section{ACKNOWLEDGMENTS}

The DMRG calculation was completed on IBM RISC6000 at International Center for Theoretical Physics, Trieste. Mobility in Europe involved in this research project was partly sponsored by EEC under contract ERB CHR XCT 940438. One of us (MF) acknowledge partial support by INFM, project HTSC. The research of MO and IA was supported in part by NSERC of Canada.

[1] A.O. Gogolin, Ann. Phys. (Paris) 19, 411 (1994)

[2] S. Tarucha, T. Honda and T. Saku, Sol. State Comm. 94, 413 (1995).

[3] K. Moon, C.L. Kane, S.M. Girvin and M.P.A. Fisher, Phys. Rev. Lett. 71, 4381 (1993).

[4] C.L. Kane and M.P.A. Fisher, Phys. Rev. Lett. 68, 1220 (1992).

[5] S. Eggert and I. Affleck, Phys. Rev. B 46, 10866 (1992).

[6] I. Affleck and W.W. Ludwig, J. Phys. A 27, 5375 (1994).

[7] C.L. Kane and M.P.A. Fisher, Phys. Rev. B 46, 15233 (1992).

[8] S. Qin, M. Fabrizio, and Lu Yu, Phys. Rev. B 54, 9854 (1996).

[9] Y. Oreg and A.M. Finkel'stein, Phys. Rev. B 53, 10928 (1996).

[10] S.R. White, Phys. Rev. Lett. 69, 2863 (1992), Phys. Rev. B 48, 10345 (1993).

[11] H.W.J. Blöte et al. Phys. Rev. Lett. 56, 742 (1986).

[12] I. Affleck, Phys. Rev. Lett. 56, 746 (1986).

[13] J.L. Cardy, Nucl. Phys. B270,186 (1986).

[14] J.L. Cardy, Nucl. Phys. B324, 581 (1989).

[15] P.W. Anderson, Phys. Rev. Lett. 18, 1049 (1967).

[16] H. Bethe, Z. Phys. 71, 205 (1931).

[17] C. Hamer, J. Phys. A19, 3335 (1986); N. M. Bogoliubov, A. G. Izergin and V. E. Korepin, Nucl. Phys. B275, 687 (1986); H. J. de Vega and M. Karowski, Nucl. Phys. B280, 619 (1987); F. Woynarovich, H.-P. Eckle and T. T. Troung, J. Phys. A22, 4027 (1989).

[18] J. des Cloizeaux and J.J. Pearson, Phys. Rev. 128, 2131 (1962).

[19] R.J. Baxter, Ann. Phys. (N.Y.) 70, 193, 323(1972).

[20] M. Fabrizio and A.O. Gogolin, cond-mat/9702080
[21] A. Furusaki, cond-mat/9702195

[22] A. Furusaki and N. Nagaosa, Phys. Rev. B 47, 4631 (1993).

TABLE I. Bulk quantities: site energy $e_{0}$, chemical potential $\mu$, spin velocity $v_{s}$, boson radius $R$, and forward scattering phase shift for boundary conditions $b=0,1: \delta$, for quarter filling $S=1 / 2$ XXZ chains with $a=0.5$ and $a=-0.5$. Values are obtained independently from DMRG (RG) and Bethe Ansatz (BA) calculations.

$\begin{array}{ccccc}a & 0.5(R G) & 0.5(B A) & -0.5(R G) & -0.5(B A) \\ e_{0} & -0.220487 & -0.220487 & -0.233179 & -0.233179 \\ \mu & -1.1335 & -1.13349 & -0.32120 & -0.321201 \\ v_{s} & 0.833 & 0.832900 & 0.500 & 0.500854 \\ 4 \pi R^{2} & 1.20 & 1.18383 & 0.72 & 0.717842 \\ \frac{\delta}{\pi}(b=1) & 0 & 0 & 0 & 0 \\ \frac{\delta}{\pi}(b=0) & -0.29 & N / A & -0.19 & N / A\end{array}$

TABLE II. Conformal field theory prediction of $d_{i}$ for $\mathrm{PBC}$ and $\mathrm{OBC}$ fixed points (see definition in Eq. 3.5 and predictions in Eqs. (3.3. 3.4)). $\delta$ is the forward scattering phase shift. The expressions for PBC are different for $a=0.5$ and $a=-0.5$.

$\begin{array}{cccc}a & \left(\frac{1}{2}, P B C\right) & \left(-\frac{1}{2}, P B C\right) & \left( \pm \frac{1}{2}, O B C\right) \\ d_{0} & \pi R^{2}\left(\frac{\delta}{\pi}\right)^{2} & \pi R^{2}\left(\frac{\delta}{\pi}\right)^{2} & \frac{1}{16}+\pi R^{2}\left(\frac{\delta}{\pi}\right)^{2} \\ d_{1} & \pi R^{2}\left(1-\frac{\delta}{\pi}\right)^{2} & \pi R^{2}\left(1-\frac{\delta}{\pi}\right)^{2} & \frac{1}{16}+\pi R^{2}\left(1-\frac{\delta}{\pi}\right)^{2} \\ d_{2} & \frac{1}{4 \pi R^{2}}+\pi R^{2}\left(\frac{\delta}{\pi}\right)^{2} & 1+\pi R^{2}\left(\frac{\delta}{\pi}\right)^{2} & \frac{9}{16}+\pi R^{2}\left(\frac{\delta}{\pi}\right)^{2} \\ d_{3} & \frac{1}{4 \pi R^{2}}+\pi R^{2}\left(1-\frac{\delta}{\pi}\right)^{2} & 1+\pi R^{2}\left(1-\frac{\delta}{\pi}\right)^{2} & \frac{9}{16}+\pi R^{2}\left(1-\frac{\delta}{\pi}\right)^{2}\end{array}$

TABLE III. Properties of $M=0$ model.

$\begin{array}{ccc}a & .5 & -.5 \\ L\left(E_{1}-E_{0}\right) & \pi \sqrt{3} / 2 & \pi \sqrt{3} / 8 \\ e_{2}(b=0) & 3 / 2 & 3 \\ e_{2}(b=1) & 9 / 4 & 6 \\ e_{3}(b=0) & 5 / 2 & 4 \\ e_{3}(b=1) & 13 / 4 & 7\end{array}$


TABLE IV. The scaling exponent $\gamma$ for small deviation from fixed points $\left(a, b_{*}\right) . \quad \gamma>0$ and $\gamma<0$ means the energy spectrum flows away from and flows to the fixed points, respectively.

$$
\begin{array}{ccccc}
\left(a, b_{*}\right) & \left(\frac{1}{2}, 1\right) & \left(\frac{1}{2}, 0\right) & \left(-\frac{1}{2}, 1\right) & \left(-\frac{1}{2}, 0\right) \\
\gamma & 1-1 / 4 \pi R^{2} & 1-4 \pi R^{2} & 2\left(1-\frac{1}{4 \pi R^{2}}\right) & 1-4 \pi R^{2} \\
& 0.17 & -0.20 & -0.78 & 0.28
\end{array}
$$

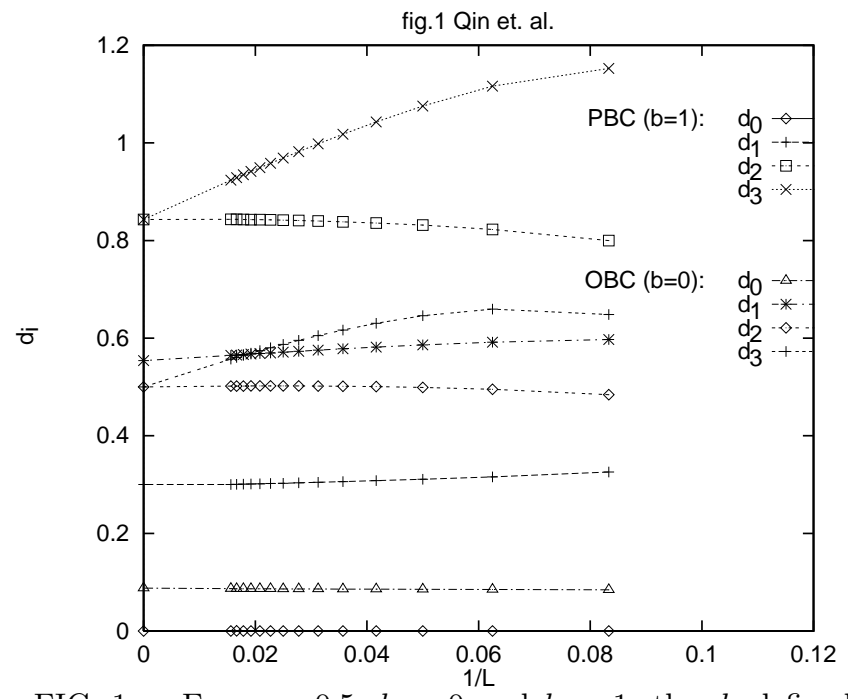

FIG. 1. For $a=0.5, b=0$ and $b=1$, the $d_{i}$ defined in Eq. 3.5) is plotted for chain lengths $L=8,16, \ldots, 64$ as a function of $1 / L$. We extrapolated the $d_{i}$ of chain lengths $L=32$ to 52 to $L \rightarrow \infty$ limit by polynomials of $1 / L$ to the second order. The extrapolated values for $d_{i}$ are in agreement with the conformal field theory predictions given in Table.II, with $\delta=0$ for $b=1$ and $\delta=-0.29$ for $b=0$.

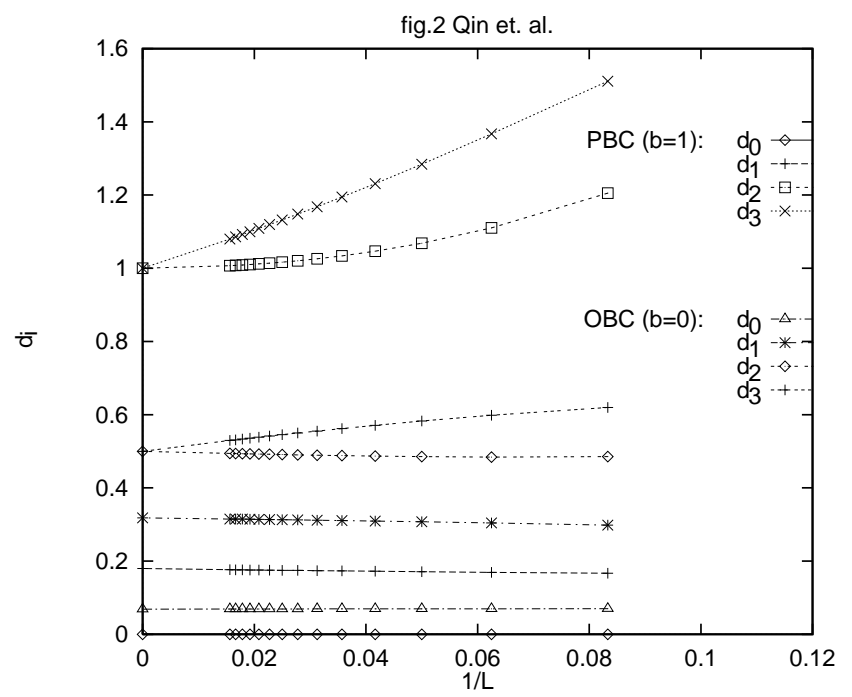

FIG. 2. For $a=-0.5, b=0$ and $b=1$, the $d_{i}$ defined in Eq. 3.5) is plotted for chain lengths $L=8,16, \ldots, 64$ as a function of $1 / L$. We extrapolated the $d_{i}$ by data of chain lengths $L=32$ to 52 to $L \rightarrow \infty$ limit by polynomials of $1 / L$ to the second order. The extrapolated values for $d_{i}$ are in agreement with the conformal field theory predictions given in Table.II, with $\delta=0$ for $b=1$ and $\delta=-0.19$ for $b=0$. 


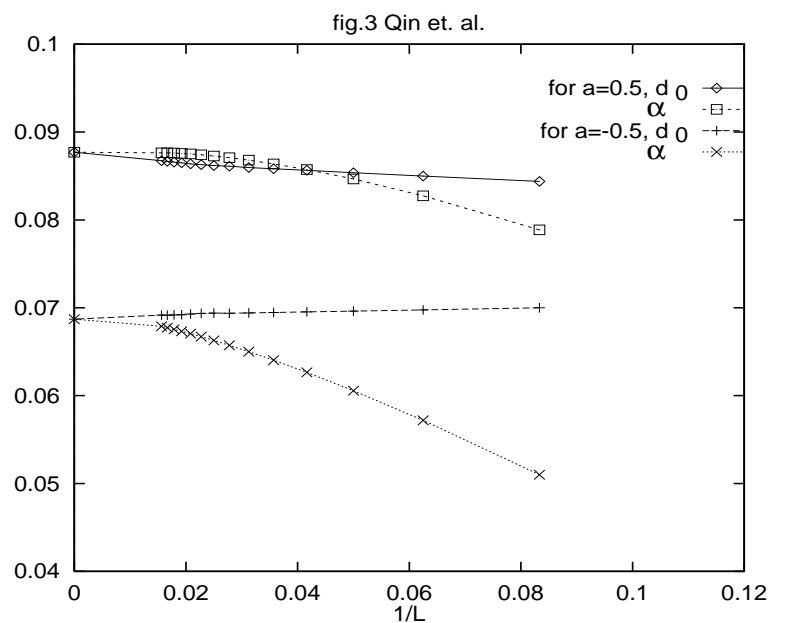

FIG. 3. The orthogonality exponent $\alpha(L)$ and ground state scaling dimension $d_{0}(L)$ for OBC chains, (see Eq.(4.7) and Eq. (4.8)), are extrapolated to $L \rightarrow \infty$ limit by polynomials of $1 / L$ to the second order. We show the extrapolated values for $\alpha$ and $d_{0}$ are equal as conformal field theory predicts.

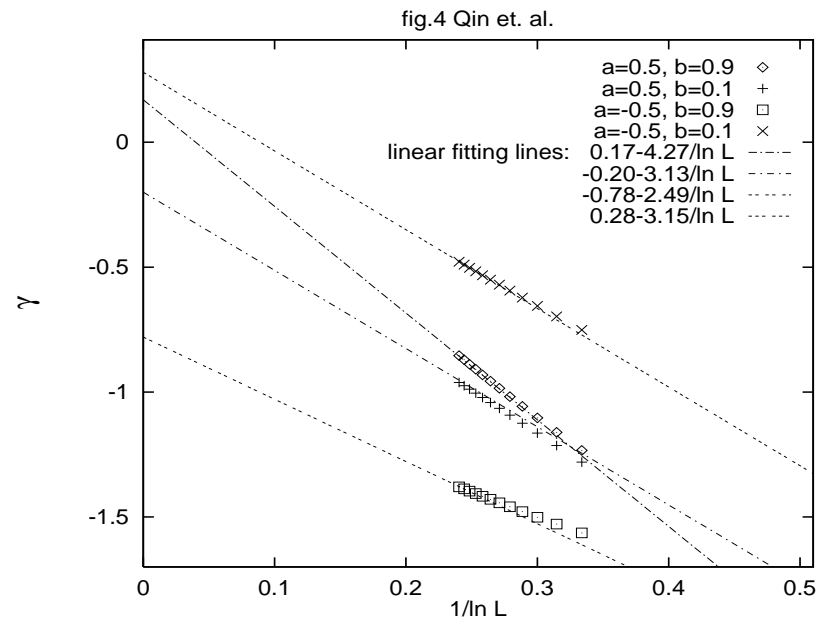

FIG. 4. The scaling exponent $\gamma$ for excitations energy near fixed points, see Eq. (4.9), is given for chains with lengths around 50. The calculated $\gamma$ are in agreement with the theoretical values listed in Table IV.

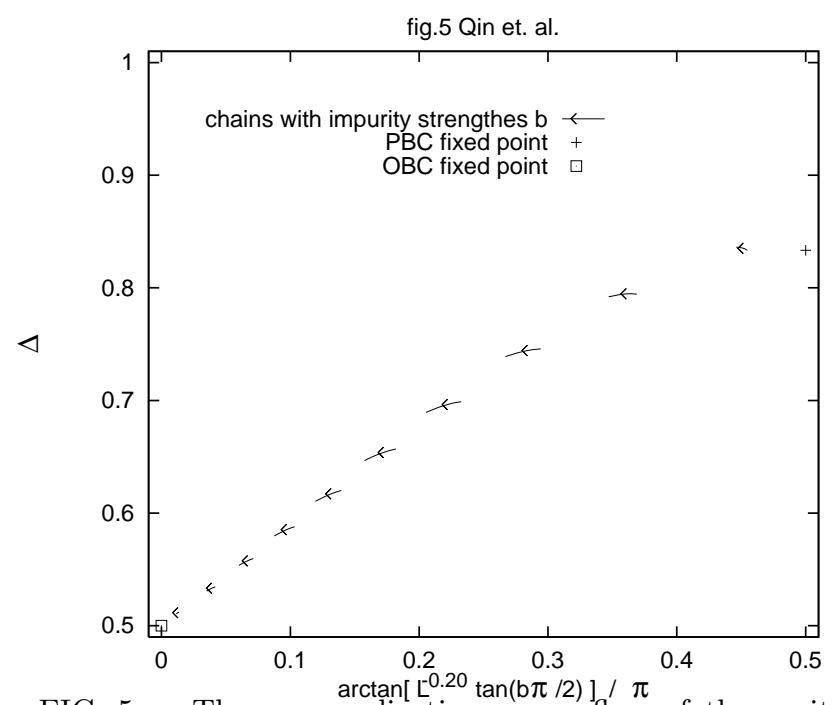

FIG. 5. The renormalization group flow of the excitation energy to $\mathrm{OBC}(b=0)$ fixed point for $a=0.5$ is qualitatively shown in this figure for the range $L=20$ to 50. The line segments from left to right of the plot are for $b=0.05,0.15, \ldots, 0.95$, respectively, and the arrow indicates how excitation energy $\Delta(0.5, b)$ flows when $L$ increases.

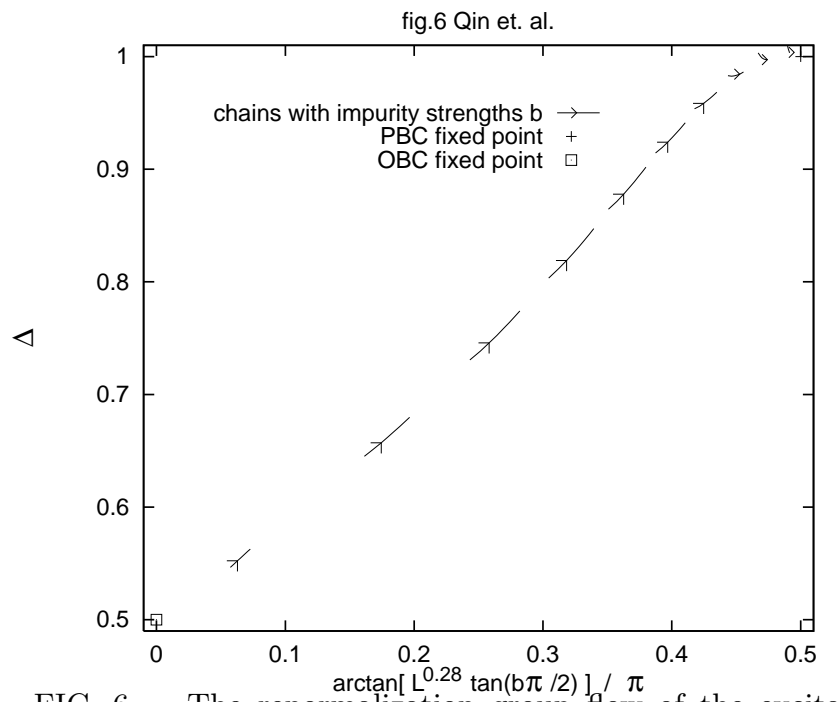

FIG. 6. The renormalization group flow of the excitation energy to $\operatorname{PBC}(b=1)$ fixed point for $a=-0.5$ is qualitatively shown in this figure for the range $L=20$ to 50. The line segments from left to right of the plot are for $b=0.05,0.15, \ldots, 0.95$, respectively, and the arrow indicates how excitation energy $\Delta(-0.5, b)$ flows when $L$ increases. 


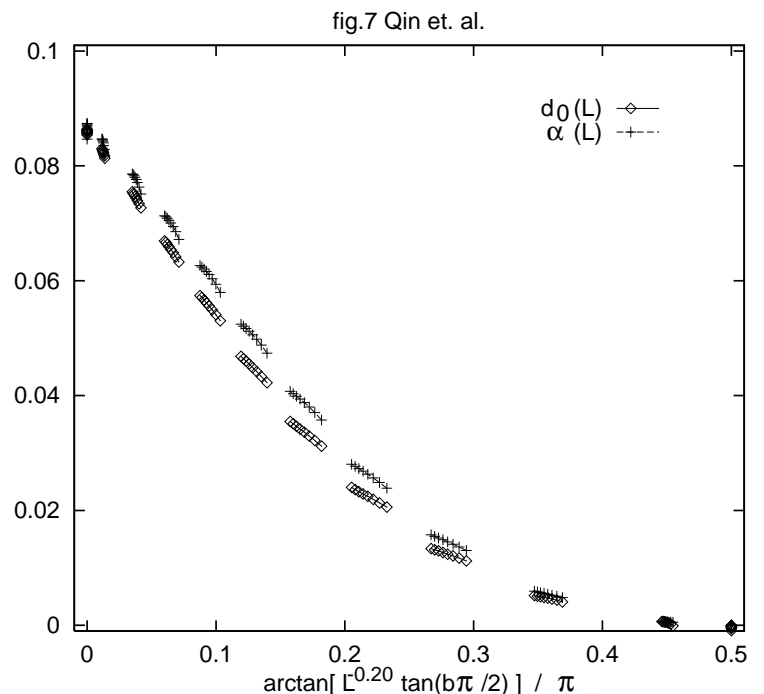

FIG. 7. The orthogonality exponent $\alpha(L)$ and ground state scaling dimension $d_{0}(L)$ for impurity chains with $a=0.5$, (see Eqs. (4.7) and Eq.(4.8), are plotted in this figure. The line segments from left to right of the plot are for $b=0.0,0.05,0.15, \ldots, 0.95,1.0$, respectively. The figure shows qualitatively how $\alpha(L)$ and $d_{0}(L)$ flow to OBC fixed point as the chain length increases.

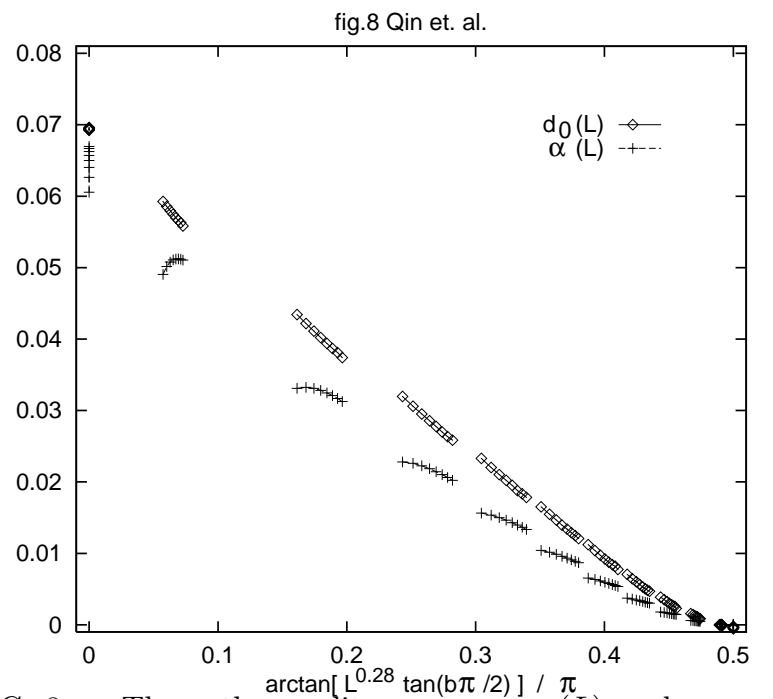

FIG. 8. The orthogonality exponent $\alpha(L)$ and ground state scaling dimension $d_{0}(L)$ for impurity chains with $a=-0.5$, (see Eqs. (4.7) and Eq. 4.8), are plotted in this figure. The line segments from left to right of the plot are for $b=0.0,0.05,0.15, \ldots, 0.95,1.0$, respectively. The figure shows qualitatively how $\alpha(L)$ and $d_{0}(L)$ flow to OBC fixed point as the chain length increases. 